Bulletin of Mathematical Biology (1999) 61, 807-828

Article No. bulm.1998.0062

Available online at http://www.idealibrary.com on IDE

\title{
Pattern Formation of Scale Cells in Lepidoptera by Differential Origin-dependent Cell Adhesion
}

\author{
TOSHIO SEKIMURA
}

College of Engineering, Chubu University,

Kasugai, Aichi 487-8501,

Japan

MEI ZHU

Department of Mathematics, Pacific Lutheran University, Tacoma, WA 98447,

U.S.A.

\section{JULIAN COOK}

Biomathematics Department, AV-633 CHS, School of Medicine,

P.O. Box 95176,

University of California,

Los Angeles, CA 90095-1766,

U.S.A.

PHILIP K. MAINI

Centre for Mathematical Biology, Mathematical Institute,

University of Oxford,

24-29 St Giles',

Oxford OX1 3LB, U.K.

\section{JAMES D. MURRAY}

Department of Applied Mathematics, University of Washington,

P.O. Box 352420,

WA 98195-2420,

Seattle, WA 98195-2420,

U.S.A.

We present a model for the formation of parallel rows of scale cells in the developing adult wing of moths and butterflies. Precursors of scale cells differentiate throughout each epithelial monolayer and migrate into rows that are roughly parallel to the body axis. Grafting experiments have revealed what appears to be a gradient of adhesivity along the wing. What is more, cell adhesivity character is maintained after grafting. Thus we suggest that it is a cell's location prior to migration that determines its interactions during migration. We use nonlinear bifurcation analysis to show that differential origin-dependent cell adhesion can result in the stabilization of rows over spots.

(C) 1999 Society for Mathematical Biology

0092-8240/99/050807+ $22 \quad \$ 30.00 / 0$

(C) 1999 Society for Mathematical Biology 


\section{INTRODUCTION}

Lepidopteran wings are covered with a large number of highly ordered scale cells. The arrangement of scale cells has a number of remarkable characteristics. Scale cells form nearly parallel rows along the anteroposterior axis of the wing (i.e., the body axis), and these rows are arranged at regular spatial intervals along the proximodistal axis. These rows are continuous across the veins and also around the two surface monolayers (i.e., the dorsal and ventral sides) of the wing.

Immediately after pupation, the epithelial cells of the wing are not differentiated and they are morphologically homogeneous. About 1 to 3 days after pupation (the timing depends on the insect and the temperature at which it develops), two cell types can be readily distinguished. The smaller cells are generalized epithelial cells (GECs) of the wing, and the larger cells are scale precursor cells (SPCs) that differentiate from GECs at the inception of adult development. SPCs are arranged in space such that they are separated from each other by GECs. Within a few hours of differentiation of the isotropically arranged SPCs, these cells become polarized along the proximodistal axis of the wing and begin to align into rows parallel to the anteroposterior axis of the wing. This row formation continues until a stable spatial periodicity of rows is established (Fig. 1). These parallel rows of SPCs that are established at the beginning of adult development maintain their arrangement throughout adult development and represent the same rows of scales that appear on the surface of the adult wing (Nijhout, 1980; Nardi and Magee-Adams, 1986; Yoshida, A. and K. Aoki, 1989).

The spatial arrangement of scale cells in periodic rows has traditionally not attracted the attention that the study of lepidopteran color patterns has received. Recently, however, progress in understanding the cellular and molecular basis of pattern formation has encouraged a fresh examination of processes involved in generating biological periodicity (Held, 1993). In the next section, we summarize what is known about the cellular and molecular processes involved in parallel row formation. In Section 3, based on experimental results on the mechanisms of row formation, we develop a mathematical model with origin-dependent adhesivity to account for the orderly rearrangement of cells during the formation of the parallel rows of scale cells. We investigate the solution properties of the model using a mixture of nonlinear bifurcation analysis and numerical simulation. The biological significance of our results is discussed in Section 4.

\section{Experimental Results on Parallel Row Formation MECHANISMS}

2.1. Cell rearrangement to form parallel rows occurs in a monolayer. There are two monolayers (upper and lower) of epithelial cells in the lepidopteran wing. During rearrangement of epithelial cells in the wing, these two monolayers are 

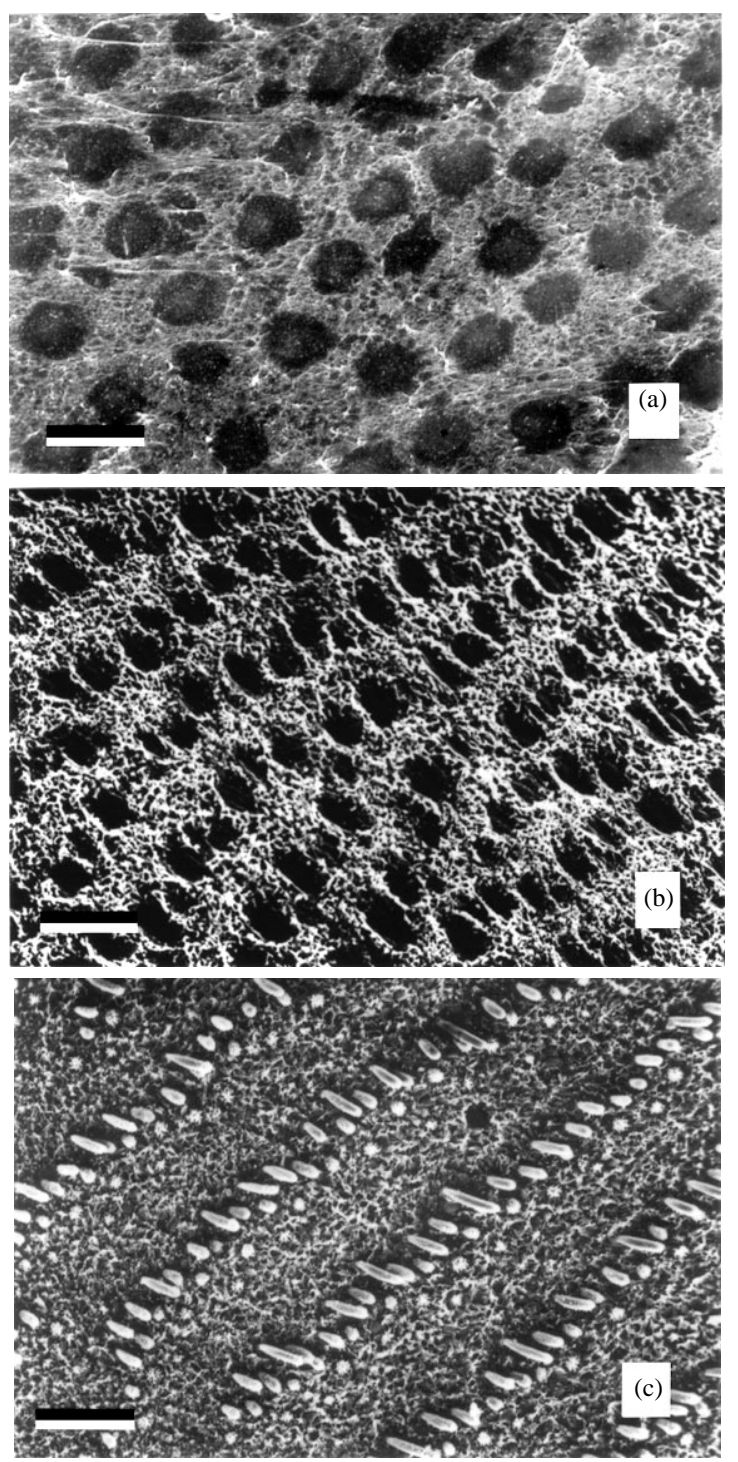

Figure 1. Parallel rows of scale cells in lepidopteran wings. (a)-(c): Surface views of the upper epithelial monolayer of the moth Mandusa wing at various times after retraction of the epithelial cells from the pupal cuticle. Proximal $\rightarrow$ distal $=$ upper left $\rightarrow$ lower right; anterior $\rightarrow$ posterior $=$ upper right $\rightarrow$ lower left. (a) At the time of epithelial retraction from the pupal cuticle ( 2.5 days after pupation), primordial scale cells (dark, circular areas) are distributed in an irregular pattern within the epithelial sheet. The cells have no obvious polarity. (b) Primordial scale cells begin to align in anastomosing rows that lie parallel to the anterior-posterior axis of the wing (3.5 days after pupation). (c) Once the alignment of cells is completed ( 5 days after pupation), scale cells begin their outgrowth in a proximal to distal direction. A stable periodicity of rows has been established [from Nardi and MageeAdams (1986) with permission, bar $=50 \mu \mathrm{m}]$. 
separated by an extracellular space. Cell rearrangement in the developing adult wing has been assumed to occur within each monolayer without influence from the other monolayer (Nardi, 1992, 1994). These findings suggest that the process of scale row formation in the lepidopteran wing does not involve complex threedimensional interactions among epithelial cells, but instead involves simpler twodimensional interactions.

2.2. Lateral inhibition probably forms the uniform pattern of SPCs. Before epithelial cells rearrange their spatial positions in the developing adult wing, SPCs differentiate from GECs. SPCs are distributed in a uniform hexagonal pattern, in which they do not come in contact with each other [see Fig. 1(a)]. Similar patterns have also been observed in other insects such as the grasshopper (Doe and Goodman, 1985) and fruitfly (Campos-Ortega, 1988). The initial isotropic spacing of SPCs is strongly suggestive of patterns generated by cells of the Drosophila integument. Bristles represent the structures of the fly integument that are developmentally equivalent to the scales of lepidopteran wings. Genetic analysis of regular bristle patterns in the integument of Drosophila has revealed most of what we know about the process (Simpson, 1990; Heitzler and Simpson, 1991; Held, 1991; Campuzano and Modolell, 1992). Bristle patterns arise in a stepwise fashion. First, a general area is specified in which all cells are competent to become bristle precursors and then one of these several competent cells is specified to be a bristle precursor cell. This cell then inhibits all nearby cells of the equivalence group from realizing their bristle cell potentialities by a process referred to as lateral inhibition (Doe and Goodman, 1985; Yoshida, A. and K. Aoki, 1989; Honda et al., 1990). The inhibitory signal emanates from the bristle precursor cell. What molecule(s) make up the inhibitory signal is still a mystery, but several loci are known to be required for transmission of the signal. Two of these loci (Notch and Delta) encode cell surface proteins and probably mediate lateral inhibition via cell contacts.

2.3. Short-range interaction mediated by differential cell adhesion. Two surface proteins (Faciclin II and neuroglian) have been found in the wing monolayers of developing adult Manduca during rearrangement of epithelial cells. The expression of these proteins changes dramatically in space and time during morphogenesis of the wing pattern. SPCs show a different pattern of protein expression from GECs during cell rearrangement (Nardi, 1992, 1994). Although neither fasciclin II nor neuroglian have been assayed for their ability to participate in heterophilic adhesive interactions, their vertebrate counterparts are known to participate in heterophilic interactions (Kadmon et al., 1990; Mauro et al., 1992). Heterophilic as well as homophilic interactions among the surface proteins expressed during cell rearrangement could provide a plethora of adhesive interactions that are instrumental in establishing the final scale patterns of lepidopteran wings. 
2.4. Origin-dependent cell adhesion. Grafting experiments within the pupal wing monolayer have been carried out to test differences in affinity properties of epithelial cells along the proximodistal axis of the wing. Those experiments have revealed that the greater the distance separating host and graft cell populations along the proximodistal axis, the more circular and constricted the interface between graft and host cells (Nardi, 1988). This result shows that affinity properties of cells depend on their positions, or more precisely, the distance between their original positions along the proximodistal axis of the wing.

2.5. Long-range interaction mediated by basal processes. During rearrangement of epithelial cells, both SPCs and GECs extend basal processes. As the alignment of SPCs into rows proceeds, extension of processes from the basal surfaces of the epithelial cells simultaneously occurs. These processes can extend for distances of several cell diameters and can establish contacts not only with adjacent cells but with cells that are four or five cell diameters away.

This long-range interaction is mediated by basal filopodia which extend over several cell diameters in many directions (Locke et al., 1981; Nardi and MageeAdams, 1986). The extension of basal processes is coincident with the surface rearrangement of cells; as soon as the SPCs have assumed their final positions in the wing monolayer and have begun to extend their polarized scale processes, all cells within the monolayer retract their basal processes.

\section{MODEL FOR SPCS WITH ORIGIN-DEPENDENT ADHESIVITY}

Based on observations on row formation, we begin by presenting a general model for aggregation in which cells move up gradients of adhesivity. As cells can respond directly to non-adjacent neighbours, we use integrals to represent the local average adhesivity to which a cell responds. In a first simplification we ignore origin-dependent effects and find that spotted or striped patterns are possible, depending on parameter values. This is based on a nonlinear bifurcation analysis, confirmed by numerical simulations. However, even when the parameter values in the model equations are such that stripes are expected it is difficult to see how such striped patterns could evolve with the predictable orientation found on lepidopteran wings. When we add origin-dependent adhesion to the basic model, further nonlinear analysis suggests that stripes predominate in a larger region of parameter space. More importantly, the stripes are more predictably aligned.

3.1. Integral representation. In this model we shall focus on only one cell type (SPCs) and assume that cells interact with each other according to the distance between their original locations (as well as the distance between their current locations).

Let $n(\boldsymbol{x}, a, t)$ denote the cell density at position $\boldsymbol{x}=(x, y)$ at time $t$ for the cells 
of a given adhesivity $a$ that originate from a position that is a distance $a$ away from the body axis (the base of the wing). We assume that cell movement is due to two processes; diffusion and advection (directed movement) in response to gradients of adhesivity. Due to the evidence for long-range interactions we shall consider a cell to respond to gradients in a spatially averaged adhesivity. As an evolution equation for cell density in space we write

$$
n_{t}=D \nabla^{2} n-\nabla \cdot[n c]
$$

where $D$ is the diffusion coefficient. The advection velocity, $\boldsymbol{c}$, is given by

$$
\boldsymbol{c}=C \nabla\left[\int n(\boldsymbol{x}-\boldsymbol{y}, a-s) w(\boldsymbol{y}, s) d s d y_{1} d y_{2}\right]
$$

where $\boldsymbol{x}=(x, y)$ and $\boldsymbol{y}=\left(y_{1}, y_{2}\right)$ are position variables, and $C$ is a positive constant. The integral represents the spatially averaged adhesivity. The degree of adhesivity as a function of distance, $\boldsymbol{y}$, and adhesivity distance (distance in adhesivity space), $s$, are incorporated in the kernel $w(\boldsymbol{y}, s)$. For simplicity, we shall suppose that this is separable (i.e., that the effects of distance in physical and adhesivity space are independent of each other). We therefore write

$$
w(\boldsymbol{y}, s)=g(\boldsymbol{y}) h(s)
$$

Suggested forms of these functions are shown in Fig. 2. We assume that $g$ displays rotational symmetry in the two spatial dimensions, and $h$ is symmetric in the adhesivity difference. Notice the threshold in adhesivity marking a transition from attraction to repulsion. Also, the spatial kernel is such that very short range attraction is weaker than middle distance attraction. This reflects the fact that scale cells appear not to come into contact with one another during reorganization. Below, we will analyse the model for the special case in which we assume that $|\boldsymbol{y}|$ and $|s|$ are both much smaller than 1. Biologically, this corresponds to the assumption that the adhesive interaction takes place over short distances in comparision to the size of the domain and that changes in the magnitude of adhesivity are very small.

To complete the model formulation we impose random initial conditions in cell density and periodic, or reflective, boundary conditions.

3.2. Mathematical analysis of the model and numerical simulations. Here we study the mathematical model proposed above for describing the mechanisms underlying the spatial patterning of scale precursor cells in lepidopteran wings. Our goal is to investigate this model mathematically and numerically to test if it generates the parallel row pattern observed in the wing. Firstly, we study a simplified model where the origin-dependent adhesion mechanism is absent. The methods we 

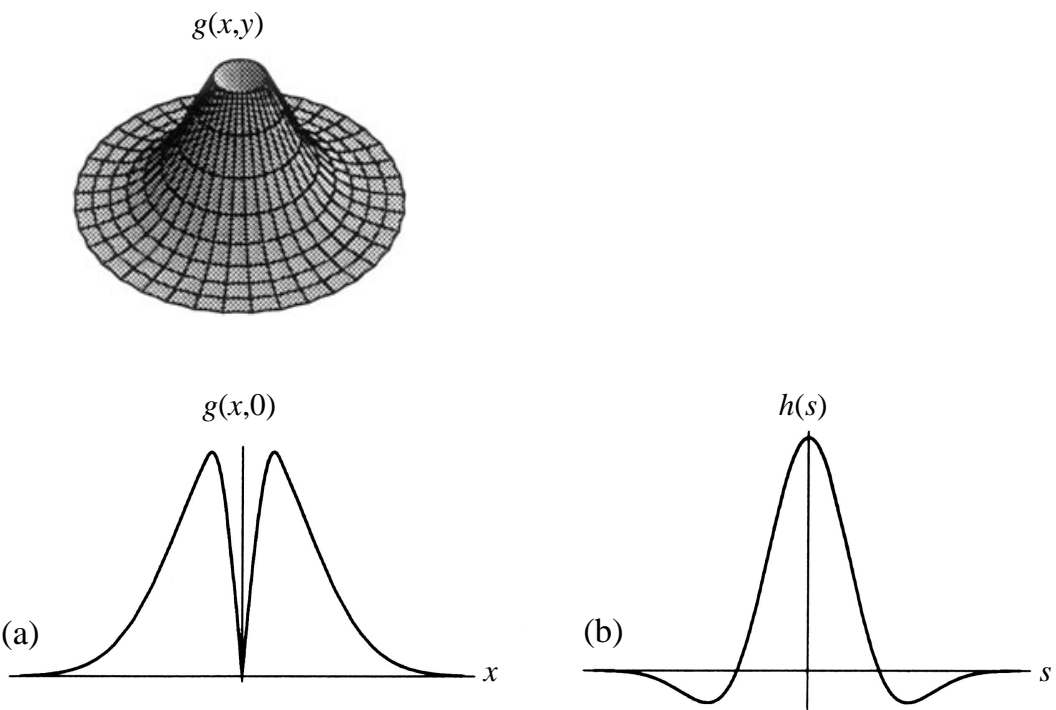

Figure 2. (a) The spatial kernel $g$. Adjacent cells are relatively unadhesive (diffusion dominates); for large distances adhesivity effects fall with distance between cells. (b) The adhesivity kernel $h$. Cells originally from nearby points attract each other. Cells of vastly different adhesivity repel each other.

use are weakly nonlinear analysis and numerical simulation. We compare our analytical results with the numerical results. Secondly, we study the effect of origindependent adhesivity on generating rows that are parallel to the body axis.

Using the assumption that $|\boldsymbol{y}| \ll 1$ and $|s| \ll 1$, we can simplify equation (1) by Taylor expanding equation (2):

$$
n_{t}=D \nabla^{2} n-C \nabla \cdot\left[n \nabla\left(n+\gamma \nabla^{2} n+\beta n_{a a}\right]+O\left(s^{4}+|\boldsymbol{y}|^{4}\right)\right.
$$

where

$$
\begin{aligned}
& \beta=\frac{1}{2} \int s^{2} h(s) d s, \\
& \gamma=\frac{1}{2} \int y_{1}^{2} g(\boldsymbol{y}) d y_{1} d y_{2} .
\end{aligned}
$$

The parameters $\beta$ and $\gamma$ are related directly to the effects of cell adhesion and distance, respectively.

In this equation, the first term on the right-hand side represents random cell motion, while the second term represents cell motion in response to adhesivity. This is a generalization of the haptotaxis term introduced by Murray and coworkers [see Murray (1993) for full details] and incorporates cell response to non-local neighbours in physical and adhesivity space. This procedure reduces the integro-partial differential equation (1) to a partial differential equation and we carry out all our analyses on the latter. 
3.2.1. Origin-independent cell adhesion.. We first consider a simple version of equation (4) in which we neglect the $O\left(s^{4},|\boldsymbol{y}|^{4}\right)$ terms, and assume that $\beta \ll \gamma$, that is,

$$
n_{t}=D \nabla^{2} n-C \nabla \cdot\left[n \nabla\left(n+\gamma \nabla^{2} n\right)\right] \text {. }
$$

Hence, this version of the model ignores the effect of the origin-dependent cell adhesion mechanism.

Linear analysis. By nondimensionalizing $n$ appropriately, we can, without loss of generality, assume that the steady state cell density is $n_{0}=1$. For linear analysis, we consider small perturbations from the uniform steady state of the form

$$
n=1+n^{\prime}
$$

where $\left|n^{\prime}\right| \ll 1$. Substituting equation (8) into (7) we obtain the linearized equation

$$
n_{t}^{\prime}=D \nabla^{2} n^{\prime}-C \nabla^{2} n^{\prime}-C \gamma \nabla^{4} n^{\prime}
$$

We look for solutions to equation (9) of the form

$$
n^{\prime}=\sum_{l=1}^{M}\left[a_{l}(t) e^{i \boldsymbol{k}_{l} \cdot \boldsymbol{x}}+\bar{a}_{l}(t) e^{-i \boldsymbol{k}_{l} \cdot \boldsymbol{x}}\right]
$$

where the bar denotes complex conjugate. Since we are interested in the initial growth of spatial pattern in cell density, we consider spatial modes, $\boldsymbol{k}_{\boldsymbol{l}}(\boldsymbol{l}=$ $1,2, \ldots, M)$, that satisfy the boundary conditions and whose corresponding amplitude functions are exponentially growing functions $\left(\left|a_{l}(t)\right|=e^{\sigma_{l} t}, \sigma_{l} \geq 0\right)$ in the short time.

We substitute (10) into (9) to obtain the dispersion relation

$$
\sigma=-(D-C) k_{l}^{2}-C \gamma k_{l}^{4}
$$

where $k_{l}=\left|\boldsymbol{k}_{l}\right|$. This is illustrated in Fig. 3 .

Thus the exponential modes $k_{l}$ in this case must satisfy $0<k_{l} \leq k_{c}=\sqrt{\frac{C-D}{C \gamma}}$. Therefore, to generate an initial growth of spatial pattern, the diffusion coefficient $D$ must be less than $C$, and there must exist at least one spatial mode $k_{l}, l=$ $1,2, \ldots, M$, that satisfies the boundary conditions and the condition $0<k_{l} \leq$ $k_{c}=\sqrt{\frac{C-D}{C \gamma}}$. This shows, as we would intuitively expect, that diffusion is the stabilizing influence, while advection is the aggregating factor.

To determine the ultimate spatial patterns generated by the full nonlinear model (7), we now investigate the behavior of the amplitude functions in large time through a weakly nonlinear analysis. 


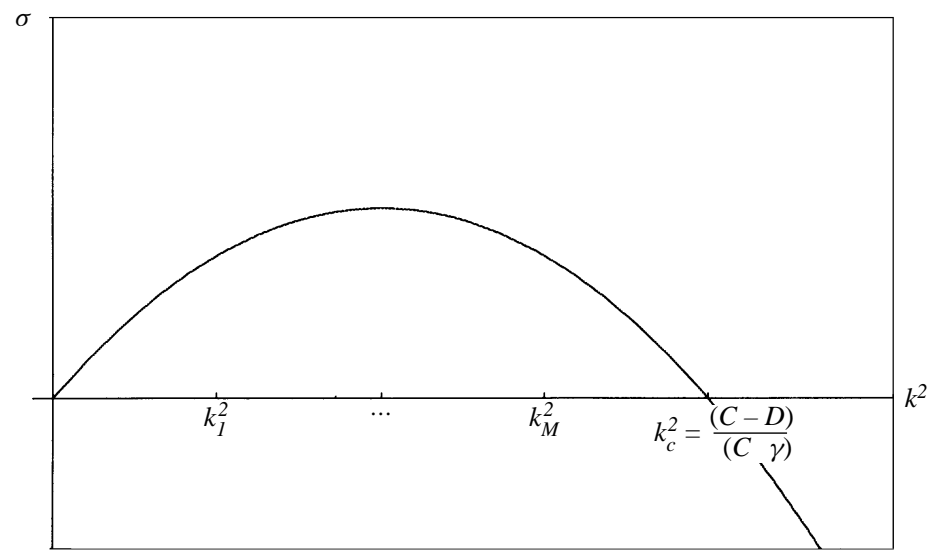

Figure 3. The dispersion relation $\left(\sigma=\sigma\left(k^{2}\right)\right)$ for the linearized version of the originindependent model (7). See text for details.

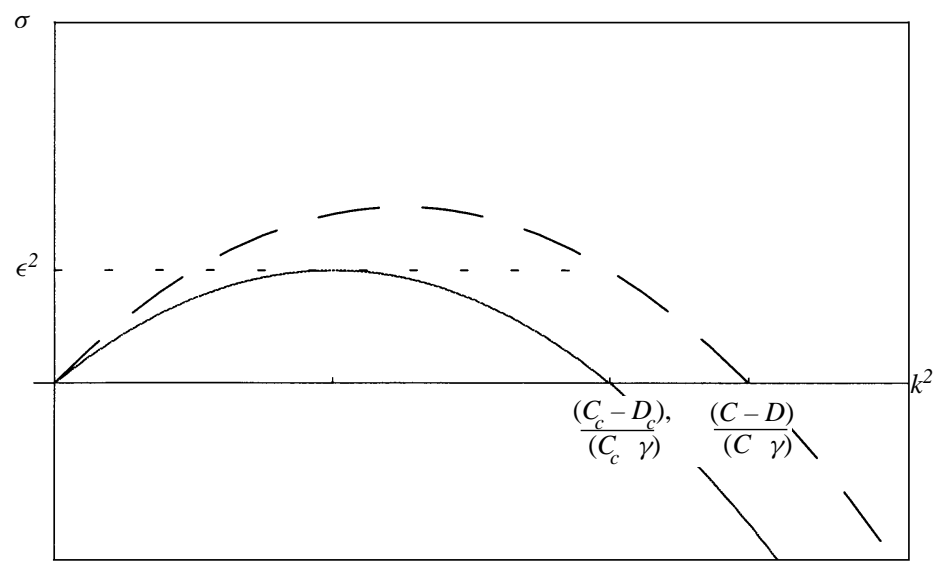

Figure 4. The effect of perturbing the parameters $D$ and $C$ around the critical values $D_{c}$ and $C_{c}$, where the dashed line is the perturbed dispersion relationship curve, and the solid line is $\left(\sigma=\sigma\left(k^{2}\right)\right)$ at critical values.

Weakly nonlinear analysis. Here we use a singular perturbation technique to study the weakly nonlinear behavior of the amplitude functions at the bifurcation point $k_{c}=\sqrt{\frac{C_{c}-D_{c}}{C_{c} \gamma}}$, where $D_{c}$ and $C_{c}$ are the critical values at bifurcation. Assume that $\boldsymbol{k}_{c l}\left(\left|\boldsymbol{k}_{c l}\right|=k_{c}, l=1,2, \ldots, M\right)$ are spatial vectors that satisfy the boundary conditions, and that there is no other spatial vector $\boldsymbol{k},|\boldsymbol{k}|<\boldsymbol{k}_{c}$, satisfying the boundary conditions. We perturb the model coefficients $D$ and $C$ around the critical values $D_{c}$ and $C_{c}$ to obtain the dispersion relation as shown in Fig. 4, where

$$
\sigma\left(k_{c}\right)=\varepsilon^{2} \ll 1 .
$$


As usual [see, for example, Fife (1979)], we consider the slow time-scale

$$
t=\frac{T}{\varepsilon^{2}}
$$

and expand $n$ with respect to $\varepsilon$ :

$$
n=1+\sum_{j=1} \varepsilon^{j} n_{j}
$$

Substituting (13) and (14) into (7) and equating powers of $\varepsilon$, we derive the following hierarchy of linear equations from which we can determine $n_{1}$ :

$O(\varepsilon)$ :

$$
L_{1} n_{1}=0,
$$

$O\left(\varepsilon^{2}\right)$ :

$$
L_{1} n_{2}=C_{c} \nabla \cdot\left[n_{1} \nabla\left(n_{1}+\gamma \nabla^{2} n_{1}\right)\right],
$$

$O\left(\varepsilon^{3}\right)$

$$
\begin{aligned}
L_{1} n_{3}= & n_{1 T}+C_{c} \nabla \cdot\left[n_{1} \nabla\left(n_{2}+\gamma \nabla^{2} n_{2}\right)\right]+C_{c} \nabla \cdot\left[n_{2} \nabla\left(n_{1}+\gamma \nabla^{2} n_{1}\right)\right] \\
& +\left(C^{\prime}-D^{\prime}\right) \nabla^{2} n_{1}+C^{\prime} \gamma \nabla^{4} n_{1}
\end{aligned}
$$

where

$$
L_{1}=\left(D_{c}-C_{c}\right) \nabla^{2}-C_{c} \gamma \nabla^{4} .
$$

By solving equation (15), we obtain $n_{1}$ in the form of

$$
n_{1}=\sum_{l=1}^{M}\left[a_{l}(T) e^{i k_{c l} \cdot x}+\bar{a}_{l}(T) e^{-i k_{c l} \cdot x}\right]
$$

where $a_{l}(T)$ are amplitude functions, $\boldsymbol{k}_{c l}$ are spatial vectors that satisfy the boundary conditions and $\left|\boldsymbol{k}_{\boldsymbol{c l}}\right|=k_{c}$. By solving (16) and applying the Fredholm alternative to (17), we obtain the Landau equations that determine the amplitude functions, $a_{l}(T)$, in (19):

$$
\frac{d A_{l}}{d T}=X_{l l} A_{l}^{2}+\sum_{j \neq l} X_{l j} A_{l} A_{j}+Y A_{j}
$$


where

$$
A_{l}=\left|a_{l}\right|^{2} \quad l=1,2, \ldots, M
$$

and $X_{l l}, X_{l j}$ and $Y$ are functions of $C^{\prime}, D^{\prime}, C_{c}, D_{c}, \gamma, \boldsymbol{k}_{c l}$ and $\boldsymbol{k}_{c j}$.

As the Landau equations (20) are the governing equations of the amplitude functions $a_{l}(T)$ on the long time-scale, we can obtain the conditions for generating certain spatial patterns by determining the stability of these amplitude functions.

For example, let us consider a special case where $M=2$ ('square' but not 'hexagonal' spots or stripes), $\boldsymbol{k}_{1}=\left(k_{c}, 0\right)$ and $\boldsymbol{k}_{2}=\left(0, k_{c}\right)$. Equation (20), in this case, reduces to

$$
\begin{aligned}
& \frac{d A_{1}}{d T}=X A_{1}^{2}+X_{12} A_{1} A_{2}+Y A_{1} \\
& \frac{d A_{2}}{d T}=X A_{2}^{2}+X_{12} A_{1} A_{2}+Y A_{2}
\end{aligned}
$$

where

$$
\begin{aligned}
X & =\frac{3 C_{c}^{2} k_{c}^{2}\left[3 k_{c}^{2} \gamma-1\right]\left[1-\gamma k_{c}^{2}\right]}{\left(C_{c}-D_{c}\right)-4 C_{c} k_{c}^{2} \gamma}, \\
X_{12} & =\frac{4 C_{c}^{2} k_{c}^{2}\left[2 k_{c}^{2} \gamma-1\right]\left[1-\gamma k_{c}^{2}\right]}{\left(C_{c}-D_{c}\right)-2 C_{c} k_{c}^{2} \gamma}, \\
Y & =2 k_{c}^{2}\left[C^{\prime}-D^{\prime}-C^{\prime} \gamma k_{c}^{2}\right],
\end{aligned}
$$

and $D^{\prime}$ and $C^{\prime}$ are, respectively, the perturbations of $\mathrm{D}$ and $\mathrm{C}$ around the bifurcation values: $D=D_{c}+\varepsilon^{2} D^{\prime}, C=C_{c}+\varepsilon^{2} C^{\prime}$.

From (22), we can obtain the steady state values of the amplitude functions and determine their linear stability using standard techniques (Jordan and Smith, 1987) (see Table 1).

From Table 1, we see that if, for example, the parameters satisfy $D_{c}<2 C_{c} / 5$ and the uniform steady state is perturbed by a small initial random perturbation, then a striped spatial pattern will be generated eventually. However, we cannot determine the direction of the stripe. In fact, Table 1 shows that the parallel and perpendicular directions are equally likely. Furthermore, it also shows that there is a parameter space in which spotted patterns are stable. 
Table 1

\begin{tabular}{lll}
\hline Steady state & $\begin{array}{l}\text { Conditions for } \\
\text { linear stability }\end{array}$ & Spatial pattern \\
\hline$\left|a_{1}\right|^{2}=\left|a_{2}\right|^{2}=0$ & $Y<0$ & None \\
$\left|a_{1}\right|^{2}=0,\left|a_{2}\right|^{2}=\frac{-Y}{X}$ & $Y>0$ and & $\begin{array}{l}\text { Stripes perpendicular to } \\
\text { the body axis }\end{array}$ \\
$\left|a_{2}\right|^{2}=0,\left|a_{1}\right|^{2}=\frac{-Y}{X}$ & $Y>0$ and & Stripes parallel to \\
& $D_{c}<2 C_{c} / 5$ & the body axis \\
$\left|a_{1}\right|^{2}=\left|a_{2}\right|^{2}$ & $Y>0$ and & Spots \\
$=-\frac{Y}{X+X_{12}}$ & $2 C_{c} / 5<D_{c}<6 C_{c} / 11$ & \\
\hline
\end{tabular}

3.2.2. Numerical simulations.. Here we numerically solve of model (7) by using a finite difference method with the aim of verifying our analytic predictions. The model parameters used in the numerical simulations are chosen to satisfy the conditions for generating (Table 1).

Simulation 1. We use a randomly distributed cell density as our initial condition and impose periodic boundary conditions. We choose $D_{c}=0.09 C_{c}$, which satisfies the condition for generating stripes given in Table 1 . The numerical results obtained are shown in Fig. 5, where the cell density distributions are shown at $t=0, t=0.05, t=0.5, t=2, t=20$, and $t=40$. The lighter color represents higher cell density while the darker color represents lower cell density. The results clearly show that a striped pattern is generated by our model equation, in accordance with the results of our weakly nonlinear analysis.

Simulation 2. Again we use a randomly distributed cell density as our initial condition, and choose $D_{c}=0.09 C_{c}$. However, in this case we impose zero-flux boundary conditions in both $x$ - and $y$-directions. The numerical results obtained are shown in Fig. 6, where the cell density distributions are shown at $t=0, t=2$, $t=12, t=20, t=30$, and $t=40$. Again we obtain a stable striped pattern.

Simulation 3. We use a randomly distributed cell density as our initial condition, and periodic boundary conditions. In this case, we use a large value for the diffusion coefficient $\left(D_{c}=0.5 C_{c}\right)$. The numerical results obtained are shown in Fig. 7, where the cell density distributions are plotted at $t=0, t=0.05, t=2$, $t=6, t=12$, and $t=28$. In this case, we obtain a stable spotted pattern, as predicted analytically (Table 1 ).

3.2.3. The effect of origin-dependent adhesivity.. The above study shows that the simple, origin-independent adhesivity model exhibits patterns of rows, parallel and perpendicular to the body axis, and spots. However, only rows parallel to the body axis are observed in lepidopteran wings. Here we investigate the effect of including origin-dependent adhesivity in the model. With the origin-dependent 

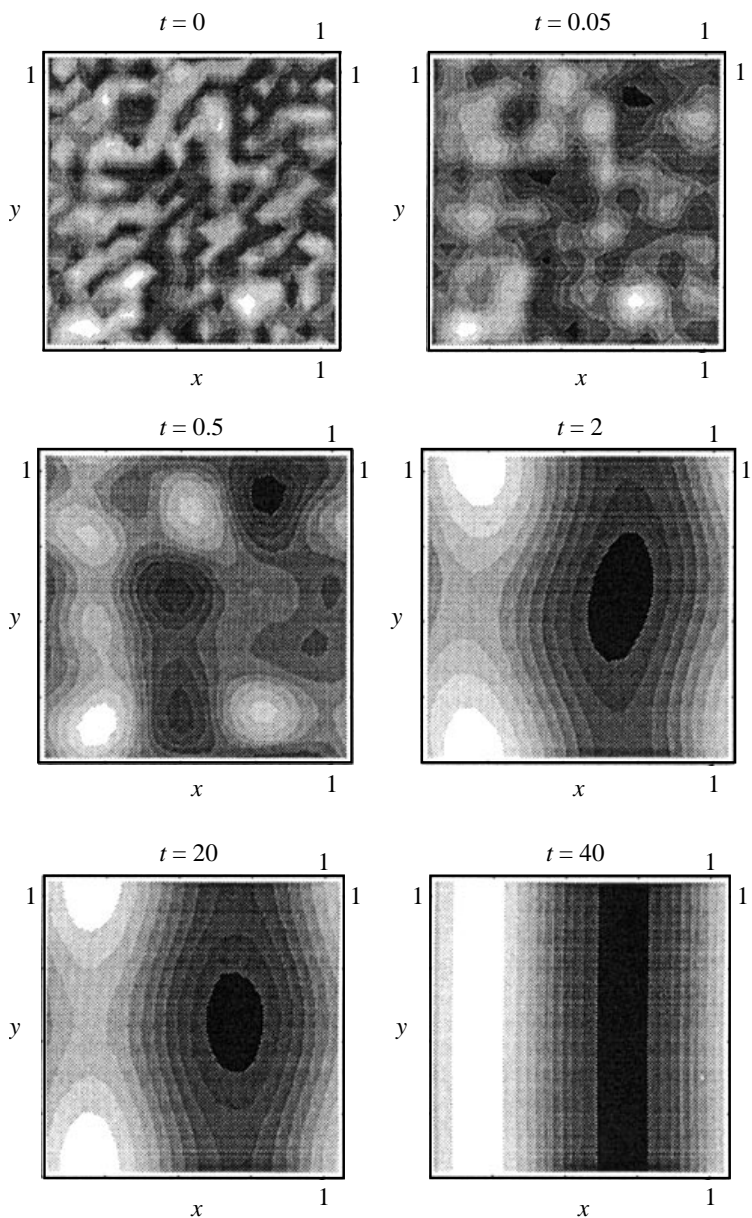

Figure 5. Results of simulation 1 , under periodic boundary conditions, where $C_{c}=$ $0.006, \epsilon=0.1, C^{\prime}=0.01, D^{\prime}=-0.001$ and $\gamma=0.0228$. Here, origin-dependent adhesivity effects are neglected.

adhesivity term the model equation becomes

$$
n_{t}=D \nabla^{2} n-C \nabla \cdot\left[n \nabla\left(n+\gamma \nabla^{2} n+\beta n_{a a}\right)\right] .
$$

Note that $n(\boldsymbol{x}, a, t)$ is the cell density at position $\boldsymbol{x}=(x, y)$ at time $t$ for the cells that originated a distance $a$ away from the body axis. We assume that $n_{a a}=E n_{x x}$, where $E$ is a proportionality constant. The motivation for making this assumption is as follows: note that $\gamma \nabla^{2} n=\gamma\left(n_{x x}+n_{y y}\right)$ is a long range diffusion term. With the origin-dependent effect, cells are less likely to diffuse in the $x$-direction (i.e., perpendicular to the body axis), so $\beta n_{a a}$ is acting as a negative diffusion term in the $x$-direction and reduces the net diffusion in the $x$-direction.

Therefore, equation (25) becomes

$$
n_{t}=D \nabla^{2} n-C \nabla \cdot\left[n \nabla\left(n+\gamma \nabla^{2} n+\beta^{*} n_{x x}\right)\right],
$$



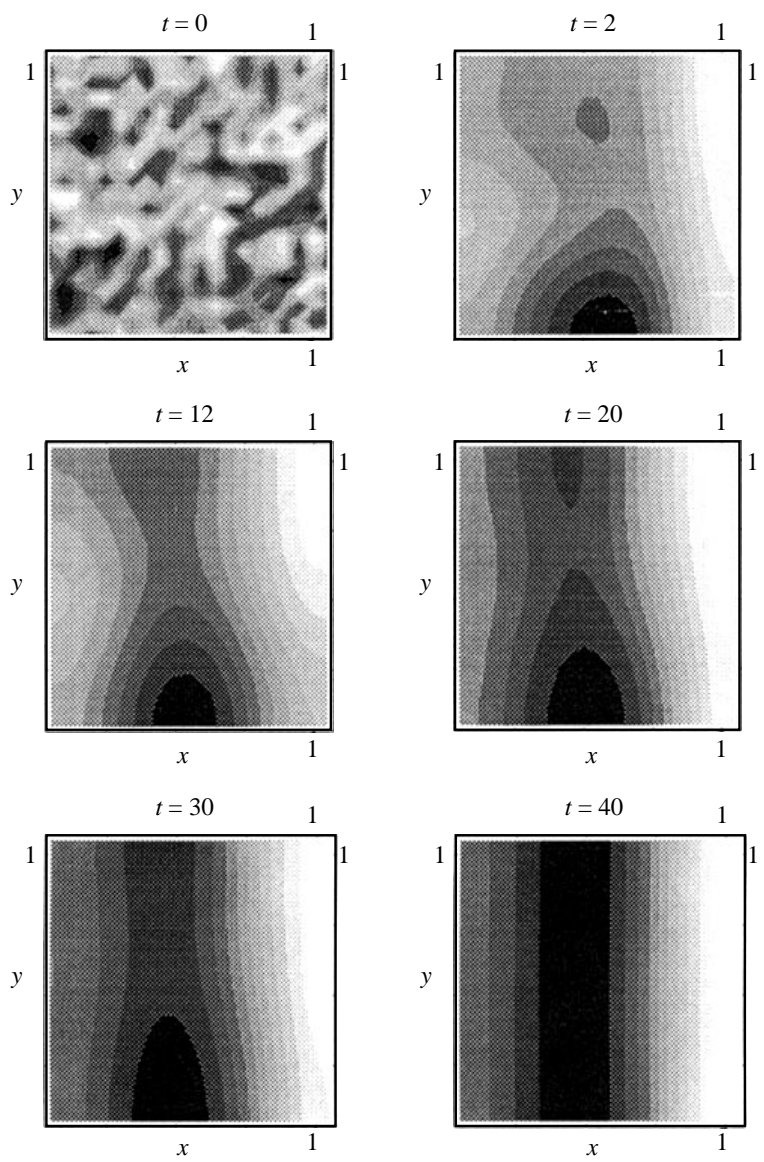

Figure 6. Results of simulation 2, under zero-flux boundary conditions. Parameter values as in the caption for Fig. 5. Again, origin-dependent adhesivity effects are neglected.

where $\beta^{*}=E \beta$. Note that although $\beta^{*}$ is negative, we assume that $\gamma+\beta^{*} \geq 0$, i.e., the effective diffusion in the $x$-direction is still positive.

Linear analysis. We linearize equation (26) around the uniform steady state $n_{0}=$ 1 , as previously, and obtain

$$
n_{t}^{\prime}=D \nabla^{2} n^{\prime}-C \nabla^{2} n^{\prime}-C \gamma \nabla^{4} n^{\prime}-C \beta \nabla^{2} n_{x x}^{\prime}
$$

where $n^{\prime}=n-1$.

We consider a square domain and restrict the analysis of this model to perturbations with spatial modes which have wave numbers of the form $\boldsymbol{k}_{1}=(k, 0)$ and $\boldsymbol{k}_{2}=(0, k)$. We look for a solution to equation (27) of the form

$$
n^{\prime}=\sum_{l=1}^{2}\left[a_{l}(t) e^{i \boldsymbol{k}_{l} \cdot \boldsymbol{x}}+\bar{a}_{l}(t) e^{-i \boldsymbol{k}_{l} \cdot \boldsymbol{x}}\right]
$$



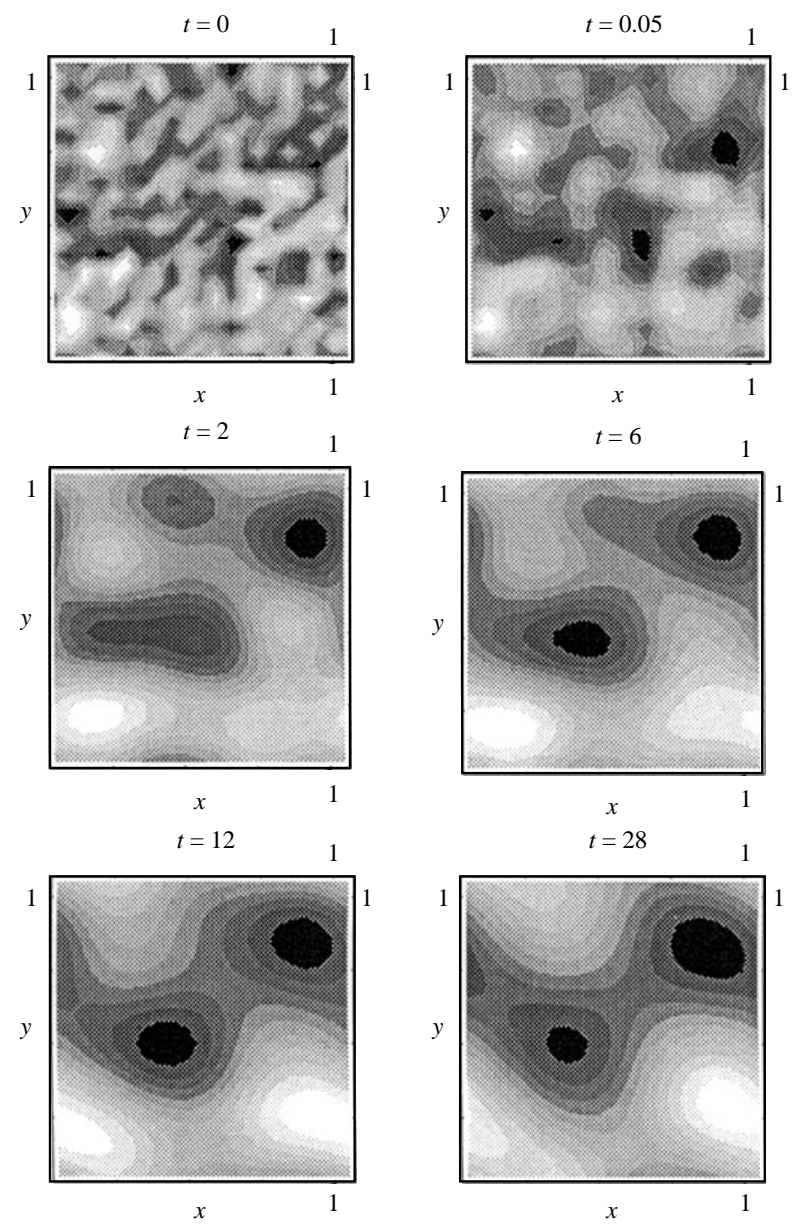

Figure 7. Results of simulation 3 , under periodic boundary conditions with large diffusion coefficient, where $C_{c}=0.006, \epsilon=0.1, C^{\prime}=0.01, D^{\prime}=-0.001$ and $\gamma=0.0127$.

$$
=a_{1}(t) e^{i k x}+a_{2}(t) e^{i k y}+\bar{a}_{1}(t) e^{-i k x}+\bar{a}_{2}(t) e^{-i k y}
$$

where $k=\left|\boldsymbol{k}_{\boldsymbol{l}}\right|, l=1,2$, and the amplitudes $a_{1}$ and $a_{2}$ have temporal growth rates $\sigma_{1}$ and $\sigma_{2}$, respectively.

Substituting equation (28) into (27), we obtain dispersion relations for $\sigma_{1}$ and $\sigma_{2}$ :

$$
\begin{aligned}
& \sigma_{1}=(C-D) k^{2}-C\left(\gamma+\beta^{*}\right) k^{4}, \\
& \sigma_{2}=(C-D) k^{2}-C \gamma k^{4} .
\end{aligned}
$$

From these equations, we note that the growth rates for the two amplitudes are different. As we assume that $\beta^{*}=\frac{1}{2} E \int s^{2} h(s) d s<0$, we have that $\sigma_{1}>\sigma_{2}$ (see Fig. 8). This means that rows parallel to the body axis are more likely to be generated than rows perpendicular to the body axis. 


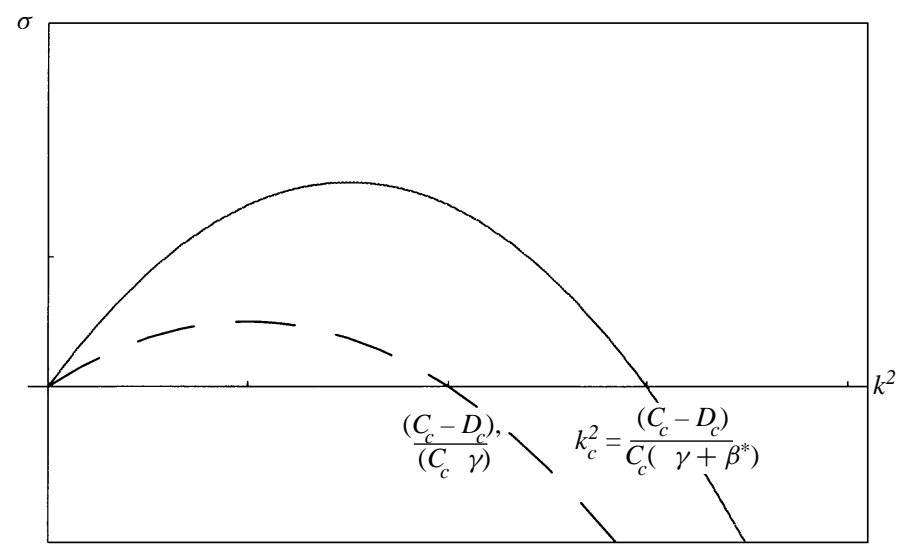

Figure 8 . The dispersion relation for the linearized version of the origin-dependent model (27). The solid curve is $\sigma_{1}=(C-D) k^{2}-C\left(\gamma+\beta^{*}\right) k^{4}$, the dashed curve is $\sigma_{2}=$ $(C-D) k^{2}-C \gamma k^{4}$.

Weakly nonlinear analysis. We now carry out a weakly nonlinear analysis on model equation (26). The procedure we use is identical to that described above for the origin-independent adhesivity model. Note, however, that in this case the bifurcation point occurs where $\sigma_{1}=0$, namely, where

$$
k_{c}^{2}=\frac{C_{c}-D_{c}}{C_{c}\left(\gamma+\beta^{*}\right)}
$$

where $D_{c}$ and $C_{c}$ are the critical parameter values for $D$ and $C$,

As before, we perturb $D$ and $C$ around the critical values of $D_{c}$ and $C_{c}$ by $\epsilon^{2} D^{\prime}$ and $\epsilon^{2} C^{\prime}$ so that

$$
\sigma_{1}\left(k_{c}\right)=\varepsilon^{2} \ll 1
$$

We again consider a slow time-scale

$$
t=\frac{T}{\varepsilon^{2}}
$$

and expand $n$ as

$$
n=1+\sum_{j=1} \varepsilon^{j} n_{j}
$$

We substitute equation (33) and (34) into (26) and equate coefficients of powers of $\varepsilon: O(\varepsilon)$ :

$$
L n_{1}=0,
$$


$O\left(\varepsilon^{2}\right)$ :

$$
L n_{2}=C_{c} \nabla \cdot\left[n_{1} \nabla\left(n_{1}+\gamma \nabla^{2} n_{1}+\beta^{*} n_{1 x x}\right)\right]
$$

$O\left(\varepsilon^{3}\right)$

$$
\begin{aligned}
L n_{3}=n_{1 T} & +C_{c} \nabla \cdot\left[n_{1} \nabla\left(n_{2}+\gamma \nabla^{2} n_{2}+\beta^{*} n_{2 x x}\right)\right] \\
& +C_{c} \nabla \cdot\left[n_{2} \nabla\left(n_{1}+\gamma \nabla^{2} n_{1}+\beta^{*} n_{1 x x}\right)\right] \\
& +\left(C^{\prime}-D^{\prime}\right) \nabla^{2} n_{1}+C^{\prime}\left(\gamma \nabla^{4} n_{1}+\beta^{*} \nabla^{2} n_{1 x x}\right),
\end{aligned}
$$

where

$$
L=\left(D_{c}-C_{c}\right) \nabla^{2}-C_{c} \nabla^{2}\left(\gamma \nabla^{2}+\beta^{*} \frac{\partial^{2}}{\partial x^{2}}\right)
$$

The solution to equation (35) on a square domain has the form

$$
n_{1}=a_{1}(T) e^{i k_{c} x}+a_{2}(T) e^{i k_{c} y}+\bar{a}_{1}(T) e^{-i k x}+\bar{a}_{2}(T) e^{-i k y},
$$

and, using the same procedure as above, we obtain the Landau equations:

$$
\begin{aligned}
& \frac{d A_{1}}{d T}=X_{11} A_{1}^{2}+X_{12} A_{1} A_{2}+Y_{1} A_{1} \\
& \frac{d A_{2}}{d T}=X_{22} A_{2}^{2}+X_{12} A_{1} A_{2}+Y_{2} A_{2}
\end{aligned}
$$

where

$$
\begin{aligned}
A_{1}(T) & =\left|a_{1}(T)\right|^{2}, \\
A_{2}(T) & =\left|a_{2}(T)\right|^{2}, \\
X_{11} & =\frac{3 C_{c}^{2} k_{c}^{2}\left[3 k_{c}^{2}\left(\gamma+\beta^{*}\right)-1\right]\left[1-\left(\gamma+\beta^{*}\right) k_{c}^{2}\right]}{\left(C_{c}-D_{c}\right)-4 C_{c} k_{c}^{2}\left(\gamma+\beta^{*}\right)}, \\
X_{12} & =\frac{4 C_{c}^{2} k_{c}^{2}\left[k_{c}^{2}\left(2 \gamma+\beta^{*}\right)-1\right]\left[1-\left(\gamma+\beta^{*}\right) k_{c}^{2}\right]}{\left(C_{c}-D_{c}\right)-2 C_{c} k_{c}^{2} \gamma-C_{c} \beta^{*} k_{c}^{2}}, \\
X_{22} & =\frac{3 C_{c}^{2} k_{c}^{2}\left[3 k_{c}^{2} \gamma-1\right]\left[1-\gamma k_{c}^{2}\right]}{\left(C_{c}-D_{c}\right)-4 C_{c} k_{c}^{2} \gamma}, \\
Y_{1} & =2\left[\left(C^{\prime}-D^{\prime}\right) k_{c}^{2}-C^{\prime}\left(\gamma+\beta^{*}\right) k_{c}^{4}\right], \\
Y_{2} & =2\left[\left(C^{\prime}-D^{\prime}\right) k_{c}^{2}-C^{\prime} \gamma k_{c}^{4}\right] .
\end{aligned}
$$

The linear stability conditions for these steady states can be easily found (see Table 2). 
Table 2 .

\begin{tabular}{lll}
\hline Steady state & $\begin{array}{l}\text { Conditions for } \\
\text { linear stability }\end{array}$ & Spatial pattern \\
\hline$\left|a_{1}\right|^{2}=\left|a_{2}\right|^{2}=0$ & $Y_{1}, Y_{2}<0$ & None \\
$\left|a_{1}\right|^{2}=0,\left|a_{2}\right|^{2}=\frac{-Y_{2}}{X_{22}}$ & Impossible & $\begin{array}{l}\text { Rows perpendicular } \\
\text { to the body axis }\end{array}$ \\
$\left|a_{2}\right|^{2}=0,\left|a_{1}\right|^{2}=\frac{-Y_{1}}{X_{11}}$ & $Y_{1}>0$ & $\begin{array}{l}\text { Rows parallel } \\
\text { to the body axis }\end{array}$ \\
$\left|a_{1}\right|^{2}=\frac{Y_{1} X_{22}-Y_{2} X_{12}}{X_{22} X_{11}-X_{12}^{2}}$ & Impossible when & Spots \\
$\left|a_{2}\right|^{2}=\frac{Y_{2} X_{11}-Y_{1} X_{12}}{X_{22} X_{11}-X_{12}^{2}}$ & $\beta^{*}<-\gamma\left(\frac{D_{c}}{C_{c}} C^{\prime}-D^{\prime}\right) /\left(C^{\prime}-D^{\prime}\right)$ & \\
\hline
\end{tabular}

From Table 2, we see that striped patterns can only occur in the direction that is parallel to the body axis under the effect of origin-dependent adhesivity. We also note that as long as the effect of origin-dependent adhesivity is sufficiently strong $\left(\beta^{*}<-\gamma\left(\frac{D_{c}}{C_{c}} C^{\prime}-D^{\prime}\right) /\left(C^{\prime}-D^{\prime}\right)\right)$, spotted patterns cannot be generated.

\section{SUMMARY AND DisCUSSION}

Several models have been proposed for spatial pattern formation in biology [for a review, see Murray (1993)]. The vast majority of these models consist of coupled systems of partial differential equations and they have been extensively studied and shown to exhibit a vast range of spatial patterns. In this paper, we have considered pattern formation of scale cells in lepidopteran wings based on a number of key biological observations, we have developed a novel model for pattern formation consisting of only one equation, of integro-partial differential type. We have shown that a simple version of this model can exhibit stripes or spots, but that a more complicated version can exhibit only stripes of a specific orientation that is consistent with biological observations.

We have assumed that the arrangement of scale precursor cells in lepidopteran wings proceeds temporally stepwise and consists of two different pattern forming processes, that is, uniform pattern formation of SPCs as the first step, noted in Section 2 [see also Fig. 1(a)] and as the second step, the formation of parallel rows (Sekimura and Yoshida, 1990). In relation to parallel row formation of scale cells, two other problems of note still remain.

The first concerns the mechanisms that generate the uniform pattern of SPCs prior to parallel row formation. It has been suggested that the isotropic spacing pattern of SPCs can be generated through lateral inhibition-a type of cell-cell interaction whereby a cell that adopts a particular fate inhibits its immediate neighbours from doing likewise. The transmembrane proteins Notch and Delta (or their homologues) have been identified as mediators of the interaction-Notch as receptor, Delta as its ligand on adjacent cells. Recently, a simple and general mathematical model of such contact-mediated lateral inhibition has been presented, based 
on the Delta-Notch mechanism of lateral inhibition (Collier et al., 1996). In our numerical simulations in Section 3, we have not used a uniform pattern of SPCs as initial condition, but instead have imposed a randomly distributed cell density of SPCs. However, it should be noted that these random perturbations can be made extremely small without affecting the results of the simulations, and they reflect the fluctuations that one would expect in a biological system of this type. The second problem is cell proliferation during the arrangement pattern formation. SPCs continue to differentiate from generalized epithelial cells during cell rearrangement. The increase in populations of SPCs is around 20\% of the total cell population. The exact value depends on the insect (Yoshida, A. and K. Aoki, 1989; Yoshida, 1993). Honda et al. (1990) have estimated, computationally, neuroblast numbers in insect neurogenesis using the lateral inhibition hypothesis of cell differentiation. We have not included cell proliferation in the model. It would result in a production term which would not affect the movement dynamics and would therefore have only a quantitative influence on the patterning behavior of the model and the main results of this paper would still hold.

Arrangement of cells into rows also occurs in the formation of pigment patterns in, for example, alligators, snakes, and fish skin. The models proposed for these types of pattern (Murray et al., 1990; Murray and Myerscough, 1991; Kondo and Asai, 1995; Varea et al., 1997) are of coupled partial differential equation type and they can exhibit a wider variety of pattern.

One of the key questions in biological pattern formation is how does a particular pattern develop in a robust and reliable manner? For example, reaction diffusion systems (Turing, 1952), can exhibit spots or stripes on two-dimensional domains. It has been shown that the form of the nonlinearity is crucial in determining which of these patterns stabilizes (Ermentrout, 1991; Lyons and Harrison, 1991; Nagorcka and Mooney, 1992; Zhu and Murray, 1995a,b). More recently, Varea et al. (1997), have shown that stripe selection and orientation can be controlled by imposing very special boundary conditions and spatially varying parameters. In this paper, we have provided a novel mechanism (origin-dependent adhesivity), for selecting the formation of stripes over spots, and orienting the stripes in the correct direction, without imposing special boundary conditions.

\section{ACKNOWLEDGEMENTS}

We would like to thank Dr J. Nardi of University of Illinois at Urbana-Champaign for his critical reading of the manuscript. This work (JDM) was supported in part by grants from the U.S. National Science Foundation (DMS-9500766) and the National Institute of Health (2P41-RR-01243-12). 


\section{REFERENCES}

Bard, J. B. L. (1981). Atitle model for generating aspects of zebra and other mammalian coat patterns. J. Theor. Biol 93, 363-385.

Campos-Ortega, J. A. (1988). Cellular interaction during early neurogenesis of Drosophila melanogaster. Trends Neurosci 11, 400-405.

Campuzano, S. and J. Modolell (1992). Patterning of the Drosophila nervous system: the achaete-scute gene complex. Trends Genetics 8, 202-208.

Collier, J. R., N. A. M. Monk, P. K. Maini and J. H. Lewis (1996). Pattern formation by lateral inhibition with feedback: a mathematical model of Delta-Notch intercellular signalling. J. Theor. Biol. 183, 429-446.

Doe, C. Q. and C. S. Goodman (1985). Early events in insect neurogenesis, II The role of cell interactions and cell lineage in the determination of neuronal precursor cells. Dev. Biol. 111, 206-219.

Ermentrout, B. (1991). Stripes or spots? Nonlinear effects in bifurcation of reactiondiffusion equations on the square. Proc. R. Soc. Lond. Ser. A 434, 413-417.

Fife, P. (1979). Mathematical Aspects of Reacting and Diffusing Systems Lecture Notes in Biomathematics, 28, Springer-Verlag.

Heitzler, P. and P. Simpson (1991). The choice of cell fate in the epidermis of Drosophila. Cell 64, 1083-1093.

Held, L. I. (1991). Bristle patterning in Drosophila. Bioessays 13, 633-640.

Held, L. I. (1993). Models for Embryonic Periodicity, Basel: Karger.

Honda, H., M. Tanemura and A. Yoshida (1990). Estimation of neuroblast numbers in insect neurogenesis using the lateral inhibition hypothesis of cell differentiation. Development 110, 1349-1352.

Jordan, D. W. and P. Smith (1987). Nonlinear Ordinary Differential Equations, Oxford: Oxford University Press.

Kadmon, G., A. Kowitz, P. Altevogt and M. Schachner (1990). The neural cell adhesion molecule N-CAM enhances L1-dependent cell-cell interactions. J. Cell Biol. 110, 193208.

Locke, M. and P. Huie (1981). Epidermal feet in insect morphogenesis. Nature 293, 733735.

Lyons, M. J. and L. G. Harrison (1991). Stripes and spots and isolated structures: the pattern-forming abilities of diverse non-linearities in reaction-diffusion mechanisms, NWPMB workshop, Vancouver B.C.

Lyons, M. J. and L. G. Harrison (1992). Stripe selection: an intrinsic property of some pattern-forming models with nonlinear dynamics. Dev. Dyn. 195, 201-215.

Kondo, S. and R. Asai (1995). A reaction-diffusion wave on the skin of the marine angelfish Pomacanthus. Nature 376, 765-768.

Kopell, N. and L. N. Howard (1973). Horizontal bands in the Belousov reaction. Science 180, 1171-1173.

Maini, P. K. and J. D. Murray (1988). A nonlinear analysis of a mechanical model for biological patternformation. SIAM. J. Appl. Math. 48, 1064-1072. 
Mauro, V. P., L. A. Krushel, B. A. Cunningham and G. M. Edelman (1992). Homophilic and heterophilic binding activities of $\mathrm{Nr}-\mathrm{CAM}$, a nervous system cell adhesion molecule. J. Cell Biol. 119, 191-202.

Murray, J. D. (1980). Lecture Notes in Biomathematics, Berlin, Heidelberg, New York: Springer, pp. 360-399.

Murray, J. D. (1981). A pre-pattern formation mechanism for animal coat markings. $J$. Theor. Biol. 88, 161-199.

Murray, J. D. (1993). Mathematical Biology, Springer-Verlag.

Murray, J. D., D. C. Deeming and M. W. J. Ferguson (1990). Size dependent pigmentation pattern formation in embryos of Alligator mississippiensis: time of initiation of pattern generation mechanism. Proc. R. Soc. Lond. Ser. B 239, 279-293.

Murray, J. D and M. R. Myerscough (1991). Pigmentation pattern formation on snakes. $J$. Theor. Biol. 149, 339-360.

Nagorcka, B. N. and J. R. Mooney (1992). From stripes to spots: prepatterns which can be produced in the skin by a reaction-diffusion system. IMA J. Math. Appl. Biol. Med. 9, 249-267.

Nardi, J. B. (1988). Establishment of a two-dimensional neural network in an insect wing. Current Issues in Neural Regeneration Research, Liss A.R., 127-136.

Nardi, J. B. (1992). Dynamic expression of a cell surface protein during rearrangement of epithelial cells in the Manduca wing monolayer. Dev. Biol. 152, 161-171.

Nardi, J. B. (1994). Rearrangement of epithelial cell types in an insect wing monolayer is accompanied by differential expression of a cell surface protein. Dev. Dyn. 199, 315325.

Nardi, J. B. and S. M. Magee-Adams (1986). Formation of scale spacing patterns in a moth wing. I. Epithelial feet may mediate cell rearrangement. Dev. Biol. 116, 278-290.

Nijhout, H. F. (1980). Ontogeny of the color pattern on the wing of Precis coenia (Lepidoptera: Nymphalidae). Dev. Biol. 80, 275-288.

Oster, G. F. and J. D. Murray (1989). Pattern formation models and development. Zool. 251, 186-202.

Sekimura, T. and A. Yoshida (1990). A model for cellular pattern formation of scales in a butterfly wing. Forma 5, 73-82.

Simpson, P. (1990). Lateral inhibition and the development of the sensory bristles of the adult peripheral nervous system of Drosophila. Development 109, 509-519.

Turing, A. M. (1952). The chemical basis of morphogenesis. Phil. Trans. R. Soc. Lond. Ser. B 237, 37-72.

Varea, C., J. L. Aragón and R. A. Barrio (1997). Confined Turing patterns in growing systems. Phys. Rev. E56, 1250-1253.

Walgraef, D., G. G. Dewel and P. Borckmans (1982). Nonequilibrium phase transitions and chemical instability. Adv. Chem. Phys. XLIX, 311-355.

Yoshida, A. and K. Aoki (1989). Scale arrangement pattern in a lepidopteran wing. I. Periodic cellular pattern in pupal wing of Pieris rapae. Dev. Growth Differ. 31, 601609. 
Yoshida, A. (1993). The spatial pattern of the cell distribution and the cell number ratio determined by competition and lateral inhibition in the butterfly wing. Forma 8, 203210.

Zhu, M. and J. D. Murray (1995a). Parameter domains for generating spatial pattern: a comparison of reaction-diffusion and cell-chemotaxis models. Int. J. Bifurcation Chaos 5, 1503-1524.

Zhu, M. and J. D. Murray (1995b). Parameter domains for spots and stripes in mechanical models for biological pattern formation. J. Nonlinear Sci. 5, 317-336.

Received 18 February 1998 and accepted 10 May 1998 